(1)

\title{
Representações sociais da satisfação com a vida de idosos aposentados
}

\author{
Elaine A. Moura, ${ }^{1}$ Francisco E. F. Delgado, ${ }^{2}$ Cláudia Helena C. Mármora ${ }^{3 *}$
}

\section{Resumo}

Introdução: O processo de envelhecimento e o período da aposentadoria não ocorrem ao sujeito isoladamente. Concepções correntes da sociedade acerca de tais movimentos da vida, que são as representações sociais, interferirão diretamente na forma como os indivíduos experienciam essas fases. Por isso é importante compreender a satisfação com a vida em idosos aposentados segundo suas características sociodemográficas. Materiais e métodos: Foram avaliados 300 idosos aposentados por meio de uma entrevista estruturada e semiestruturada, que abordou as variáveis sociodemográficas e a satisfação com a vida. Foi utilizado o software Statistical Package for Social Sciences versão 20 para análise estatística. Resultados: Dos 300 idosos avaliados, 61,7\% eram do sexo feminino; a maioria estudou até o nível fundamental (61,6\%), $52 \%$ são casados ou vivem com algum companheiro(a); apresentaram idade média de 74,33 $\pm 6,61$ anos; renda média de $\mathrm{R} \$ 2317,8 \pm 2259,8$; e média de filhos de 2,12 $\pm 2,0$. Ao avaliar a satisfação com a vida nestas variáveis, a única que apresentou diferença estatística foi o número de filhos ( $p=0,00037$ ). Discussão: As representações sociais dos idosos aposentados sobre a satisfação com a vida permitiram evidenciar que está ocorrendo uma reestruturação do impacto que a aposentadoria causava na vida do indivíduo, permitindo uma superação de dificuldades psico-sócio-históricas na configuração do envelhecimento e da aposentadoria. Conclusões: A maneira como um indivíduo que envelhece consegue manter um funcionamento psicológico positivo e aumentar sua satisfação com a vida ao chegar à aposentadoria é uma questão importante para a sociedade.

Descritores: Envelhecimento; Aposentadoria; Satisfação pessoal.

\section{Abstract}

\section{Social representations of satisfaction with life in the retired elderly}

Introduction: The aging process and the retirement period are not isolated phenomena.Notions and concepts about life are social representations which will directly impact the way individuals experience these phases. For this reason, it's important to understand the satisfaction with life in elderly retirees approached according to their sociodemographic characteristics. Materials and methods: 300 retired elderly people went through a structured and semi-structured interview, which addressed sociodemographic variables and satisfaction with life. The statistical analysis of the research was done using Statistical Package for Social Sciences, version 20. Results:
1. Setor de Fisioterapia. Hospital Universitário. Universidade Federal de Juiz de Fora. Juiz de Fora, MG, Brasil.

2. Departamento de Psicologia. Instituto de Ciências Humanas. Universidade Federal de Juiz de Fora. Juiz de Fora, MG, Brasil.

3. Faculdade de Fisioterapia. Universidade Federal de Juiz de Fora. Juiz de Fora, MG, Brasil.

\section{*Endereço para correspondência:}

Rua Professor Clóvis Jaguaribe, 240, apto 201

Juiz de Fora, MG, Brasil. CEP: 36021-700.

E-mail: claudia.marmora@ufjf.edu.br

Revista HUPE, Rio de Janeiro, 2018;17(2):7-15

Recebido em 07/05/2018. Aprovado em 31/08/2018.

Of the 300 elderly people evaluated, $61.7 \%$ were female; the majority stayed in school only up to middle school (61.6\%), $52 \%$ are married or live with a partner; had an average age of $74.33 \pm 6.61$ years; average income was $\mathrm{R} \$ 2317.8 \pm 2259.8$; and average number of children was $2.12 \pm 2.0$. When assessing life satisfaction according to these variables, the only statistically significant difference was the number of children ( $\mathrm{p}$ $=0.00037$ ). Discussion: Social representations of the retired elderly about their satisfaction with life have made it possible to show that a restructuring of the impact that retirement has on an individual's life is taking place. This change can allow to overcome psycho-socio-historical difficulties of aging and retirement. Conclusions: The way aging individuals manage to maintain a positive psychological performance and increase their satisfaction with life upon retirement is an important issue for society.

Keywords: Aging; Retirement; Personal satisfaction.

\section{Resumen}

\section{Representaciones sociales de la satisfacción con la vida de ancianos jubilados}

Introducción: Las representaciones sociales sobre la jubilación pueden interferir directamente en la forma en que los individuos experimentan esas fases. Por eso, es importante tener una satisfacción con la vida de un anciano revelador de sus dos características sociodemográficas. Materiales y métodos: Se evaluaron 300 ancianos jubilados por medio de una entrevista estructurada y semiestructurada, que abordó las variables 


\section{Artigo original}

sociodemográficas y la satisfacción con la vida. El análisis estadístico fue realizado por medio del software Statistical Package for Social Sciences, versión 20. Resultados: De los 300 ancianos evaluados, el 61,7\% eran del sexo femenino; la mayoría estudió hasta el nivel fundamental (61,6\%), el 52\% está casado o vive con algún compañero (a); presentaron una edad promedio de $74,33 \pm 6,61$ años; el ingreso promedio de $\mathrm{R} \$ 2317,8 \pm 2259,8$; y el promedio de hijos de 2,12 $\pm 2,0$. Al evaluar la satisfacción con la vida en estas variables, la única que presentó diferencia estadística fue el número de hijos ( $\mathrm{p}=$ 0,00037). Discusión: El estudio permitió evidenciar que está ocurriendo una reestruc- turación del impacto que la jubilación causaba en la vida de un individuo, permitiendo una superación de las dificultades psico-socio-histórica en la configuración del envejecimiento y la jubilación. Conclusiones: La manera como un individuo que envejece logra mantener un funcionamiento psicológico positivo y aumentar su satisfacción con la vida al llegar a la jubilación, se constituye como una cuestión importante para la sociedad.

Palabras clave: Envejecimiento; Jubilación; Satisfacción personal.

\section{Introdução}

Devido à crescente esperança de vida e taxas de fecundidade em declínio, a proporção de pessoas acima de 60 anos está aumentando mais rápido do que qualquer outro grupo etário. ${ }^{1}$ As projeções indicam que em 2050 a população brasileira será de 253 milhões de habitantes. ${ }^{2}$ O envelhecimento humano é considerado parte do ciclo de vida, e nele emergem experiências e características peculiares resultantes da trajetória e da formação do indivíduo idoso. ${ }^{3}$

A configuração do desenvolvimento humano é expressa de forma psico-sócio-histórica, três prismas que integram em uma mesma perspectiva o humano enquanto membro da espécie humana (corpo e mente) e como participante de um processo histórico (ser biológico e social). ${ }^{4} \mathrm{O}$ papel social é um pressuposto importante nessa formação, pois decorre do estilo de vida vivenciado e das condições atuais. Dentro deste contexto, destaca-se a aposentadoria, momento no qual o indivíduo se distancia da vida produtiva. ${ }^{5}$

Com o afastamento da atividade laboral observase, geralmente, uma diminuição no círculo de amizades vinculadas ao trabalho, pois os idosos tendem a diminuir suas relações sociais e a não exercerem a mesma participação social. ${ }^{6}$ O convívio, os relacionamentos interpessoais e a troca de experiências, antes possibilitadas pelo trabalho, são então substituídas muitas vezes pelo isolamento e pela ociosidade, o que pode marcar a velhice com sentimentos de inutilidade produtiva e de incapacidade para o estabelecimento de novas relações. Além disso, é nesta fase que ocorrem mais frequentemente as perdas de parentes e amigos. Em contrapartida, a aposentadoria possibilita a convivência mais intensa com a família, a qual poderá ser harmoniosa ou conflituosa. ${ }^{5,6}$

O processo de envelhecimento e o período da aposentadoria não ocorrem ao sujeito isoladamente, pois concepções correntes da sociedade acerca de tais movimentos da vida irão interferir diretamente na forma como os indivíduos experienciam essas fases. Se esse processo de transição for marcado pela noção de declínio e improdutividade, isso pode apresentar consequências graves para o desenvolvimento humano das gerações atuais e futuras, bem como para o desenvolvimento social e econômico do país e das organizações de trabalho?

Todas essas mudanças podem alterar a satisfação que o idoso tem com a vida devido ao estereótipo negativo ainda associado ao envelhecimento e à aposentadoria. O tema satisfação com a vida em idosos tem sido amplamente discutido e pesquisado desde o início deste século. Tal construto é determinado pela realização de nossas necessidades, desejos e vontades, sendo o resultado de uma comparação entre as nossas próprias expectativas e o que realmente conseguimos. ${ }^{8}$

Felizmente, as conotações negativas ligadas ao envelhecimento estão perdendo forças, principalmente pela quantidade crescente de pesquisas em gerontologia acerca de um envelhecimento saudável, pelas preocupações com a saúde das pessoas mais velhas e pelas construções de novas representações sociais da velhice., ${ }^{2,9}$

O estudo das representações sociais busca compreender como pessoas comuns, comunidades e instituições produzem saberes sobre si, sobre os outros e sobre os objetos sociais que lhe são relevantes. As representações sociais focalizam os processos envolvidos nesta interação social, possibilitando que os indivíduos deem sentido a fatos novos ou desconhecidos e atribuam significado à sociedade e ao universo a que pertencem, criando um senso comum. Elas são criadas de forma interna, mental, em que o processo coletivo penetra como fator determinante dentro do pensamento individual. ${ }^{10,11}$ As pessoas 
e os grupos criam representações no decurso da comunicação e da cooperação e, uma vez criadas, elas adquirem uma vida própria; circulam, se encontram, se atraem, se repelem e se cristalizam continuamente através da fala, do gesto, do encontro no universo cotidiano, e dão oportunidade ao nascimento de novas representações. ${ }^{11}$

As representações sociais, cuja contribuição tem sido significativa para a compreensão de diversos fenômenos, são um referencial teórico importante em investigações sobre o processo de envelhecimento. Conhecer as representações sociais de alguns fatores pode auxiliar na compreensão do fenômeno do envelhecimento individual e coletivo, já que o envelhecimento humano é considerado um termômetro do progresso e desenvolvimento socioeconômico de um país. Portanto, esta pesquisa caracterizará as representações sociais da satisfação com a vida de idosos aposentados em diversas estratificações, quanto ao gênero, à escolaridade, ao estado civil, à idade, à renda e ao número de filhos.

\section{Materiais e métodos}

A população foi de indivíduos com 65 anos ou mais, de ambos os sexos residentes na zona urbana da cidade de Juiz de Fora, Minas Gerais, Brasil. Este município possui 16 unidades territoriais demarcadas a partir de critérios de localização geográfica e nível socioeconômico. $\mathrm{O}$ tamanho amostral por gênero e faixa etária, para um erro amostral de 5\%, foi de 385 indivíduos. Na primeira etapa, foram selecionadas, aleatoriamente, cinco entre as 16 unidades (unidades 1, 4, 10, 11 e 13). Em seguida, foram sorteados os bairros em cada unidade e ruas dos bairros selecionados. As unidades territoriais são compostas por bairros subdivididos em setores censitários, que são formados por ruas definidas em quarteirões específicos. Foi preservada a proporcionalidade de idosos em cada região nos diferentes bairros visitados. Para a seleção, foi adotado o procedimento porta-a-porta, foram visitadas as casas de cada rua, em uma ordem previamente determinada, até que a meta para aquela localidade fosse atingida. A coleta ocorreu durante o período entre janeiro de 2009 e janeiro de 2010, realizada por estudantes, devidamente treinados, dos cursos de fisioterapia, de medicina e de psicologia da UFJF, totalizando 461 indivíduos entrevistados e 427 analisados, pois 34 foram excluídos. ${ }^{12}$ Como a pesquisa atual abordará os aposentados, a amostra será composta por 300 idosos.
Os critérios de exclusão adotados foram: estar acamado; residir em instituições de longa permanência; morar na zona rural; ser portadores de incapacidade como sequelas graves de acidente vascular encefálico; imobilidade; doença de Parkinson avançada; apresentar pontuação menor que 14 no Miniexame do Estado Mental (MEEM); não ser aposentado.

Os participantes preencheram voluntariamente um termo de consentimento informado e foram respeitadas as regras da Comissão Nacional de Ética em Pesquisa do Ministério da Saúde (CONEPE/MS - 313/2008). Este trabalho contou com o apoio do Conselho Nacional de Desenvolvimento Científico e Tecnológico (CNPq) sob o processo número 555087/2006-9.

A pesquisa foi realizada por meio de entrevista com questões estruturadas e semiestruturas em que foram abordadas as seguintes variáveis:

a) Ogênero foi registrado como variável categórica, masculino $\mathrm{x}$ feminino.

b) A idade foi registrada como variável contínua e depois tabulada em faixas de 65 a 69,70 a 74,75 a 79 e 80 anos e mais.

c) A escolaridade foi registrada como variável categórica e foi obtida por meio da pergunta "Até que ano de escola o (a) Sr (a) estudou?". As respostas da questão incluíam as seguintes alternativas:

(1) Nunca foi à escola (nunca chegou a concluir a $1^{\underline{a}}$ série primária ou o curso de alfabetização de adultos)

(2) Curso de alfabetização de adulto

(3) Primário (atual nível fundamental, $1^{\underline{a}}$ a $4^{\mathrm{a}}$ série)

(4) Ginásio (atual nível fundamental, $5^{\mathrm{a}}$ a $8^{\mathrm{a}}$ série)

(5) Científico, clássico (atuais curso colegial ou normal, curso de magistério, curso técnico)

(6) Curso superior

(7) Pós-graduação, com obtenção do título de Mestre ou Doutor.

Os registros sobre o nível de escolaridade foram depois transformados em quatro faixas correspondendo as alternativas: baixa (nunca foi à escola e curso de alfabetização de adultos); fundamental (primário e ginásio); médio (científico, clássico) e superior (curso superior e pós-graduação).

d) A renda familiar foi registrada como variável contínua e obtida por meio da pergunta "Qual a renda mensal da sua família, ou seja, das pessoas que moram em sua casa, incluindo o(a) senhor(a)?" Os valores da renda foram tabulados em faixas de salários mínimos $(\mathrm{SM}) . \leq 1 ; 1,1$ a 3,0; 3,1 a 5,0; 5,1 a 10,0 e >10 salários mínimos mensais. 


\section{Artigo original}

e) O número de filhos foi obtido com a pergunta "Quantos filhos o(a) Sr/Sra tem?" Foi registrada como variável contínua e tabulada em quantidade de filhos. 1 = não tem filhos; $2=1$ a 4 filhos; $3=5$ a 9 filhos e $4=$ $\geq 10$ filhos.

f) O estado civil foi alistado como variável categórica e foi obtido pela questão "Qual é o seu estado civil?" com as seguintes opções de resposta:

(1) Casado(a) ou vive com companheiro (a)

(2) Solteiro(a)

(3) Divorciado(a) / Separado(a)

(4) Viúvo(a)

g) A aposentadoria foi registrada como variável categórica com a pergunta “O(a) senhor(a) é aposentado(a)?", apresentando como respostas (1) Sim e (2) Não.

h) A satisfação com a vida foi obtida por meio da pergunta "O senhor(a) está satisfeito(a) com sua vida hoje?" A resposta era dada sobre uma escala de 3 pontos em que 1 = pouco; 2 = mais ou menos; 3 = muito.

A análise dos dados foi realizada pelo software Statistical Package for Social Sciences (SPSS) versão 20.0. As variáveis do estudo não respeitaram os critérios de normalidade e homogeneidade de variância, observados, respectivamente, pelos testes de Kolmogorov-Smirnov e Levenne, optando desta forma pela utilização de testes não-paramétricos. Para as variáveis categóricas (gênero, escolaridade e estado civil) foram utilizados a frequência absoluta, a frequência relativa e o teste do Qui - quadrado $\left(X^{2}\right)$. Para as variáveis contínuas (idade, renda e número de filhos), média e desvio padrão. Para analisar a relação entre a aposentadoria e a satisfação com a vida foram utilizados os testes de Mann-Whitneye Kruskall-Wallis/ post-hoc de Hochberg nas seguintes estratificações: gênero, idade, escolaridade, renda familiar, estado civil e número de filhos.

\section{Resultados}

A idade média dos 300 idosos investigados foi de $74,33 \pm 6,61$ anos, sendo a maioria do sexo feminino. A renda média encontrada foi de 2317,8 $\pm 2259,8$ reais, sendo que a maioria recebe renda de até três salários mínimo, 54,7\%. A média de filhos foi de 2,12 $\pm 2,0$, assim a faixa de 1 a 4 filhos apresentaram a maior frequência com 63,3\%. As características quanto ao gênero, ao estado civil e à escolaridade podem ser observadas na tabela 1 .

Foram comparadas as frequências de resposta de satisfação com a vida entre os idosos aposentados em relação as variáveis categóricas, e percebeu-se que não foram observadas diferenças significativas entre grupos referentes ao estado civil $(p=0,927)$, à escolaridade $(p$ $=0,779)$ e ao gênero $(p=0,570)$. Em relação ao estado civil, os idosos casados ou que vivem com algum companheiro(a) apresentaram maior frequência de resposta de alta satisfação quando comparados aos separados e aos divorciados. Quanto à escolaridade, os idosos do grupo do nível fundamental apresentaram maior frequência de respostas de satisfação com a vida quando comparados àqueles com maior nível de instrução. E ao gênero, o feminino apresentou uma frequência maior de satisfação com a vida quando comparado ao masculino, principalmente quanto à alternativa "muito". Todos estes dados podem ser observados na figura 1 .

Ao analisar as frequências de respostas de satisfação com a vida entre os idosos aposentados em relação as variáveis contínuas, observou-se que houve diferença estatisticamente significativa apenas em relação à quantidade de filhos $\left(X^{2}=8,48 ; g l=3 ; p=0,00037\right)$ tabela 2, mostrando que aqueles idosos que têm entre 1 a 4 filhos encontram-se mais satisfeitos com a vida quando comparado aos outros, figura 2. Quanto à idade, a distribuição de frequência de satisfação ocorreu de forma homogênea; e em relação à renda familiar, mesmo não observada diferença estatística, percebeu que houve uma maior frequência de resposta de satisfação com a vida entre aqueles que recebem de 1,1 a 3,0 salários mínimos, e que esta frequência diminuiu à medida que a renda aumentava.

\section{Discussão}

Ao avaliar a representação social da satisfação com a vida em idosos aposentados, por meio das variáveis estudadas, percebemos que a temática envelhecimento aborda diversas questões e que este período da vida não pode ser analisado como um fenômeno homogêneo e generalizável. Em relação ao gênero, não foi observada diferença estatística entre idosos aposentados, pressupondo que este processo de mudança já está sendo bem aceito por homens e mulheres. Houve, porém, maior frequência de respostas de maior satisfação no sexo feminino.

Tal fato pode ser justificado pela maior quantidade de idosas no estudo, já que o período do envelhecimento passa por um processo de feminização. ${ }^{13}$ Este mesmo dado já foi constatado no estudo de Angeli et al em que a população feminina apresentou maior satisfação com a vida durante o envelhecimento. ${ }^{14}$ Isso vai ao encontro 
de representações sociais do envelhecimento, que o envelhecer feminino está mais relacionado com a vida doméstica e os laços familiares, e que o envelhecimento masculino está mais atrelado à questão funcionalista, que fica comprometida com a aposentadoria, agravada com a perda do trabalho., ${ }^{2,3}$

Em relação ao estado conjugal, houve predominância de idosos casados ou que vivem com algum(a) companheiro(a), totalizando 52\% dos entrevistados, sendo estes os que apresentaram maiores índices de satisfação com a vida. No estudo de Angelini et al também foi observado que a presença de algum companheiro eleva a satisfação com a vida de idosos, pois reduz o processo de isolamento e sensação de vazio. Por isso é importante que os indivíduos idosos tenham contato frequente com outros idosos ou membros do grupo

Tabela 1. Características dos idosos aposentados em termos de gênero, estado civil e escolaridade

\begin{tabular}{|c|c|c|c|c|c|}
\hline & & \multicolumn{2}{|c|}{ Participantes (300) } & \multirow[b]{2}{*}{$x^{2}$} & \multirow[b]{2}{*}{$p$} \\
\hline & & $\mathrm{n}$ & $\%$ & & \\
\hline Gênero & $\begin{array}{l}\text { Feminino } \\
\text { Masculino }\end{array}$ & $\begin{array}{l}185 \\
115\end{array}$ & $\begin{array}{l}61,7 \\
38,3\end{array}$ & 16,3 & $<0,001$ \\
\hline Escolaridade & $\begin{array}{l}\text { Básica } \\
\text { Fundamental } \\
\text { Colegial } \\
\text { Superior }\end{array}$ & $\begin{array}{c}32 \\
185 \\
57 \\
26\end{array}$ & $\begin{array}{c}10,6 \\
61,6 \\
19 \\
8,6\end{array}$ & 222,3 & $<0,001$ \\
\hline Estado civil & $\begin{array}{l}\text { Casado(a) ou vive com } \\
\text { companheiro(a) } \\
\text { Solteiro } \\
\text { Divorciado(a) } \\
\text { Viúvo(a) }\end{array}$ & $\begin{array}{l}156 \\
36 \\
19 \\
89\end{array}$ & $\begin{array}{c}52 \\
12 \\
6,3 \\
29,7\end{array}$ & 152,1 & $<0,001$ \\
\hline
\end{tabular}

Nota: Valores de $p$ são para demonstrar as diferenças estatísticas entre as frequências observadas e esperadas de cada variável. $\mathrm{N}$ : frequência dos participantes; \%: porcentagem.

Tabela 2. Percentuais de respostas relativas aos níveis de satisfação com a vida, conforme a idade, a renda familiar e a quantidade de filhos

\begin{tabular}{|c|c|c|c|c|c|c|}
\hline & & \multirow[b]{2}{*}{$\mathrm{n}$} & \multicolumn{3}{|c|}{ Satisfação com a vida } & \multirow[b]{2}{*}{$p$} \\
\hline & & & Pouca & Mais ou menos & Muita & \\
\hline \multirow{4}{*}{ Idade } & $65-69$ & 82 & 1,66 & 10,33 & 15,33 & \multirow{4}{*}{$0,409^{b}$} \\
\hline & $70-74$ & 87 & 1,66 & 9,00 & 18,33 & \\
\hline & $75-79$ & 65 & 1,66 & 5,00 & 15,00 & \\
\hline & $\geq 80$ & 66 & 1,66 & 8,00 & 12,33 & \\
\hline \multirow{5}{*}{$\begin{array}{l}\text { Renda familiar } \\
\text { em salários }\end{array}$} & $<1$ & 36 & 1,67 & 3,33 & 7,00 & \multirow{5}{*}{$0,860^{\mathrm{b}}$} \\
\hline & 1,1 a 3,0 & 164 & 2,66 & 18,00 & 34,00 & \\
\hline & 3,1 a 5 & 51 & 2,00 & 5,00 & 10,00 & \\
\hline & 5,1 a 10 & 36 & 0,33 & 4,00 & 7,66 & \\
\hline & $>10$ & 13 & - & 2,00 & 2,33 & \\
\hline \multirow{4}{*}{$\begin{array}{l}\text { Quantidade } \\
\text { de filhos }\end{array}$} & 0 & 34 & 0,33 & 4,67 & 6,33 & \multirow{4}{*}{$0,00037^{* b}$} \\
\hline & 1 a 4 & 199 & 5,66 & 23,00 & 37,67 & \\
\hline & 5 a 9 & 56 & 0,66 & 3,66 & 14,33 & \\
\hline & $\geq 10$ & 11 & - & 1,00 & 2,67 & \\
\hline
\end{tabular}

(b) Teste de Kruskal-Wallis/teste de Hochberg 


\section{Artigo original}

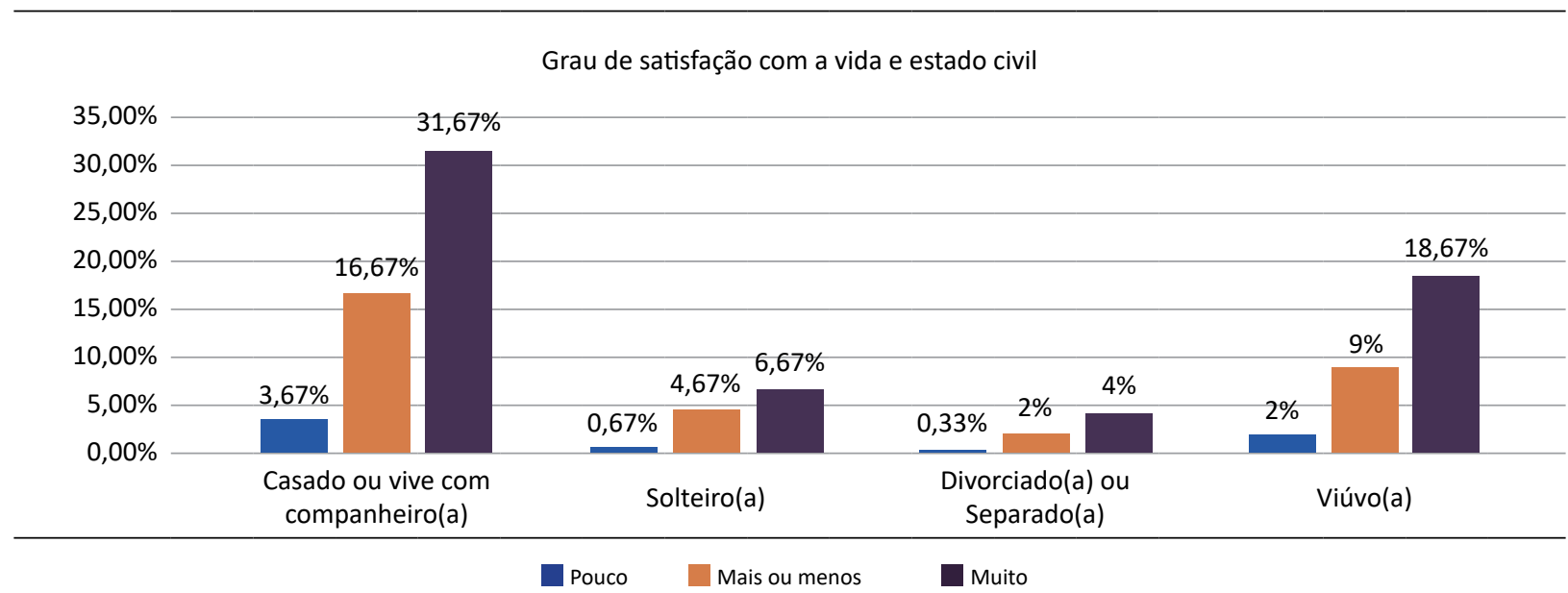

Grau de satisfação com a vida e escolaridade

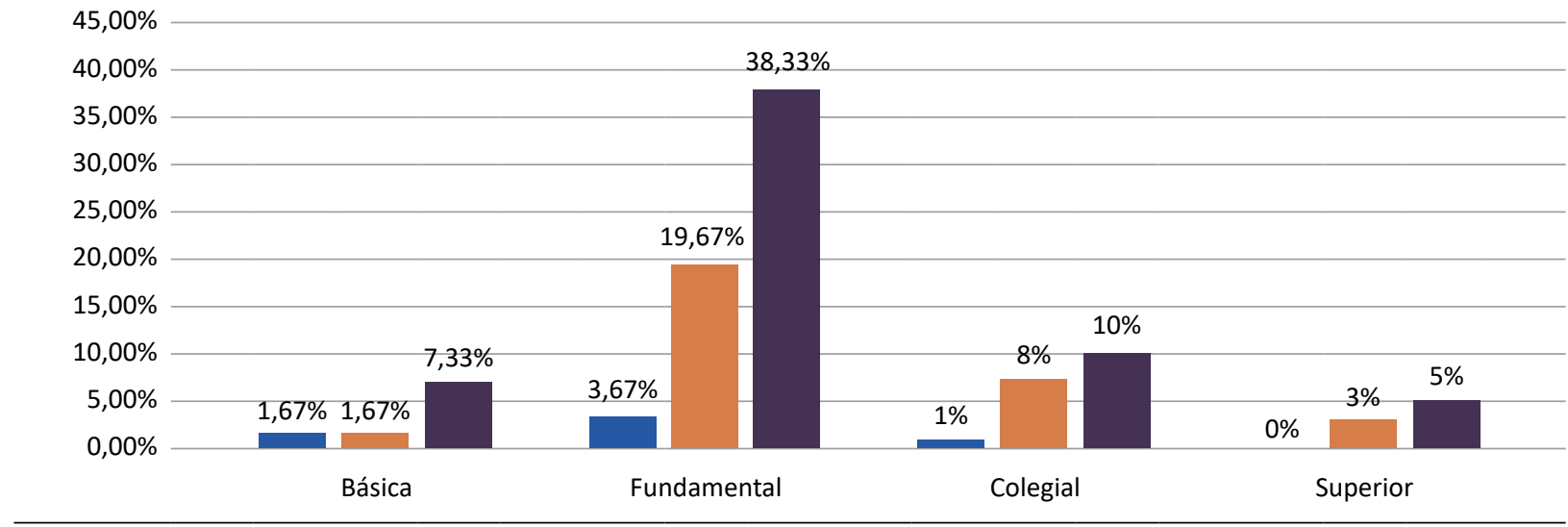

Pouco Mais ou menos $\square$ Muito

Grau de satisfação com a vida e gênero

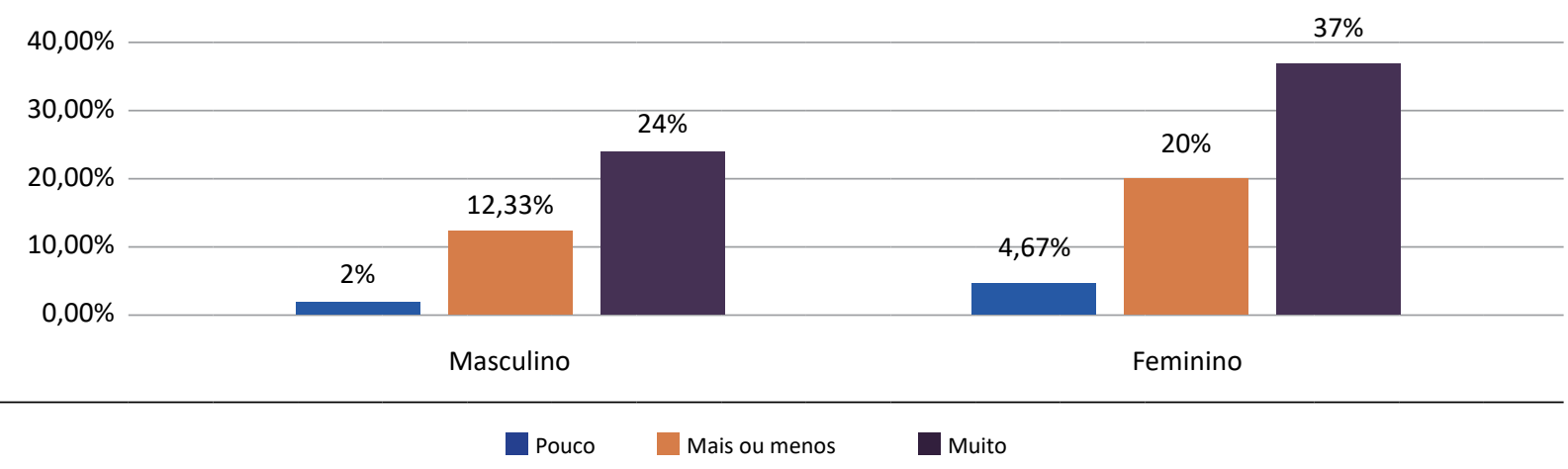

Figura 1. Percentuais de respostas relativas à satisfação com a vida comparada entre os grupos de idosos referentes aos níveis de escolaridade, do estado civil e do gênero 


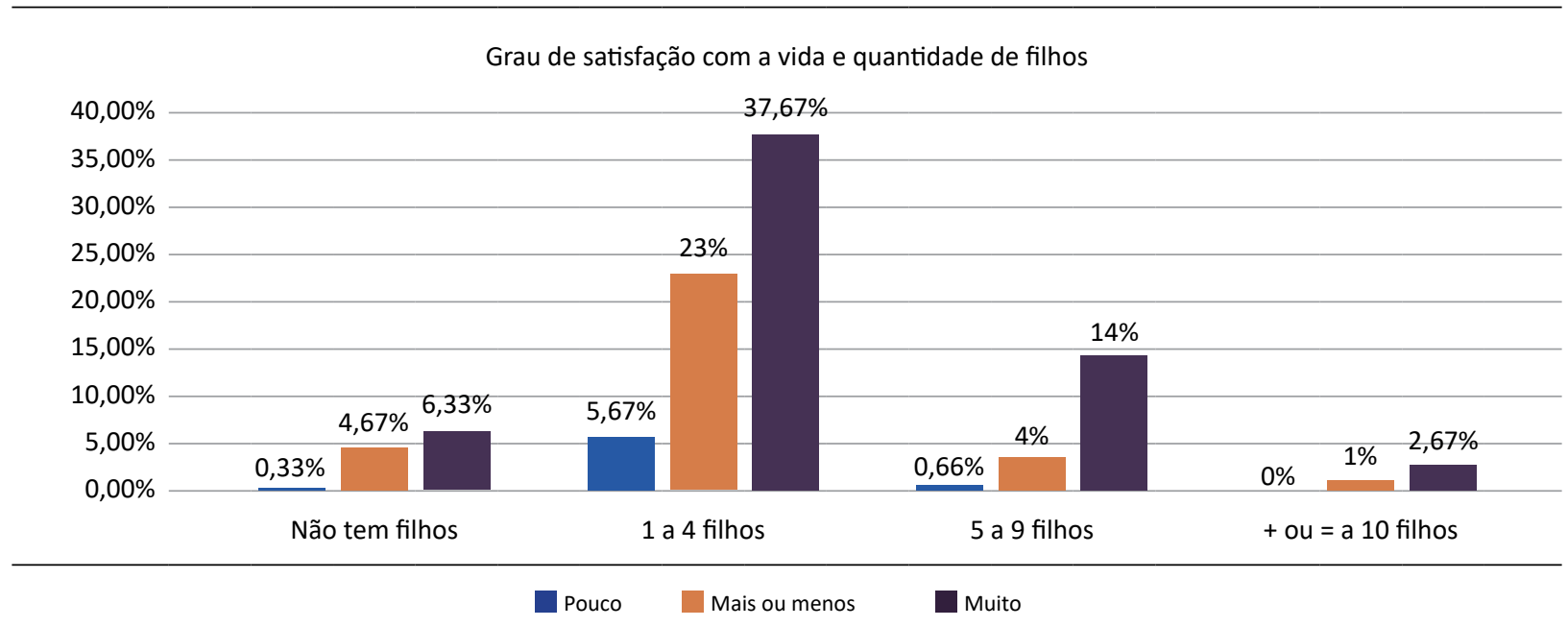

Figura 2. Distribuição das frequências de respostas da satisfação com a vida estratificada pela quantidade de filhos

que vivenciam o envelhecimento e/ou que pensam sobre o envelhecer. Destaca-se assim a importância da participação de idosos nos centros de convivência para a terceira idade, pois são locais que estimulam a pessoa idosa a exercitar suas capacidades de adaptação normativa e a aceitar suas próprias mudanças, além de manter a interação social e os vínculos de amizade. 15,16

Foi observado que $61,6 \%$ dos idosos estudaram até o nível fundamental. Essa realidade mostra a representação da menor escolaridade na população idosa, sendo importante compreender e implantar iniciativas públicas e ações não governamentais voltadas à alfabetização e à educação continuada de idosos, pois este fato pode influenciar a vida social e econômica e a busca por serviços de saúde. ${ }^{17,18}$ Porém a prevalência de um grau de instrução mais baixa não afetou a satisfação com a vida; pelo contrário: foi o grupo que apresentou a maior incidência de satisfação.

A idade é um fator importante na formação dos construtos psicológicos relacionados ao envelhecimento. O relacionamento entre idade e satisfação com a vida tende a variar de acordo com a importância de fatores socioeconômicos, culturais e políticos. Nesta pesquisa a idade não influenciou a satisfação com a vida dos idosos aposentados, quadro semelhante ao encontrado na pesquisa de Angelini et al. Isso pode ser elucidado pelo processo de reformulação que vem ocorrendo no antigo paradigma do envelhecimento. Atualmente, o envelhecimento é visto como um período de continuidade do desenvolvimento hu- mano, uma fase que pode/deve ser vivenciada de forma positiva e saudável, transmutando a imagem negativa anteriormente ligada ao processo de envelhecimento em outra relacionada à saúde, ao lazer, ao bem-estar e à qualidade de vida que proporcionam uma satisfação com a vida. ${ }^{8}$

A quantidade de filhos foi o único fator que apresentou diferença significativa em relação à satisfação com a vida na presente pesquisa, mostrando que os idosos aposentados que têm maior quantidade de filhos relataram índices menores de satisfação com a vida quando comparados aos idosos com até 4 filhos. Isso pode ser justificado pelo maior tempo despendido na criação dos filhos e menor tempo gasto com as preocupações e satisfações individuais. Além disso, os filhos constituem um forte preditor que pode ser positivo ou negativo de satisfação com a vida, principalmente de um casal. Os filhos podem atuar como um fator que reduz o rompimento ou que cause um afastamento conjugal, que pode ser mediado pela quantidade de filhos. ${ }^{19,20}$

Além disso, ter muitos filhos pode direta ou indiretamente afetar a renda familiar, principalmente quando quem sustenta a família aposenta, já que os proventos muitas vezes são menores que os salários recebidos pelos trabalhadores quando empregados. Em famílias extensas os idosos ainda contribuem para o rendimento mensal do domicílio e o sustento de filhos e netos, e em muitos casos a aposentadoria é a única renda fixa que a família possui. ${ }^{19,20}$ 


\section{Artigo original}

A aposentadoria torna-se questionável quando se verifica o rendimento mensal familiar dos chefes de família aposentados. Foi observado que em média $66,6 \%$ dos entrevistados recebem até três salários mínimos. Ao analisar os dados da pesquisa, observa-se que não houve diferença de satisfação com a vida entre os grupos de idosos, e que aqueles que recebem até 3 salários mínimos foram os que apresentaram maior frequência de satisfação com a vida. Isso também foi observado na pesquisa de Moura: os idosos de diferentes classes econômicas também não apresentaram diferença significativa quanto à satisfação com vida, mas aqueles de classe mais baixa relataram com mais frequência estarem mais satisfeitos. ${ }^{8}$ É preciso ressaltar que a pesquisa supracitada abordou a satisfação com a vida em domínios específicos, e não a satisfação global como a pesquisa atual. A avaliação da satisfação com a vida é muito subjetiva e, como tal, reflete as expressões de cada pessoa quanto aos seus próprios critérios para avaliá-la.

As representações sociais dos idosos aposentados sobre a satisfação com a vida permitiram evidenciar que está ocorrendo uma reestruturação do impacto que a aposentadoria causa na vida de um indivíduo. Vem ocorrendo uma superação das dificuldades psico-sócio-históricas na configuração do envelhecimento e da aposentadoria.

Foi possível perceber isso nos idosos aposentados, pois a satisfação deles com a vida independeu do gênero, da idade, da escolaridade, do estado civil, da renda familiar e do número de filhos dos aposentados. A maneira como um indivíduo que envelhece consegue manter um funcionamento psicológico positivo e aumentar sua satisfação com a vida ao chegar à aposentadoria é uma questão importante para a sociedade.

\section{Conclusões}

Os temas envelhecimento e aposentadoria devem ser mais discutidos, desde a escola, para mudar a visão do envelhecimento nas gerações mais novas. Também as empresas devem investir em programas de preparação para a aposentadoria, propiciando aos funcionários aumentar seu conhecimento acerca deste período e tomar decisões e fazer planejamentos e tornando a própria cultura organizacional mais sólida.

É importante a implementação de políticas que buscam instruir precocemente sobre a aposentadoria, pois neste período ocorre uma ruptura da estrutura organizacional na qual o indivíduo estava inserido. As representações sociais de idosos aposentados sobre a satisfação com a vida podem indicar trajetos para futuras intervenções em saúde física e mental e para a própria produção acadêmica sobre um envelhecimento saudável. É preciso enfatizar que a aposentadoria não deve ser vista como o fim dos projetos, mas sim como um recomeço, no qual a condição temporal foi alterada e assim precisa ser reestruturada. Existem poucas pesquisas que abordam a relação da quantidade de filhos com a satisfação com a vida de idosos, sendo um indicativo para futuras investigações.

\section{Referências}

1. OMS | Envejecimiento. WHO. [acesso em 21 abr 2018]. Disponível em: http://www.who.int/topics/ageing/es/

2. Miranda GMD, Mendes A da CG, Silva ALA da. Population aging in Brazil: current and future social challenges and consequences. Rev Bras Geriatr E Gerontol. 2016;19(3):507-19.

3. Faleiros $\mathrm{V}$. O direito humano ao envelhecimento e o impacto nas políticas públicas. Envelhecimento no Brasil: desafios e compromissos. In: Envelhecimento e Subjetividade: desafios para uma cultura de compromisso social. 1. ed. Brasília:Conselho Federal de Psicologia; 2009. p. 63-77.

4. Mármora CHC. A (a)praxia na Doença de Alzheimer. Juiz de Fora: Editora UFJF;2013.

5. van den Bogaard L, Henkens K. When is quitting an escape? How different job demands affect physical and mental health outcomes of retirement. Eur J Public Health. 2018;28(5):815-819. doi: 10.1093/eurpub/cky040.

6. Bercht AM, Scheeren E, Kunzler R, et al. Relações de gênero, trabalho e aposentadoria: Um diálogo necessário sobre a família, o cotidiano, as relações sociais e condições de vida na velhice. Semin Iniciaç Científica. 21 de outubro de 2013:122.

7. Felix YTM, Catão MFFM. Envelhecimento e aposentadoria por policiais rodoviários. Psicol Amp Soc. 2013;25(2):420-9.

8. Moura EA. Envelhecimento bem-sucedido: aspectos motores e psicológicos de idosos da cidade de Juiz de Fora. Juiz de Fora. Dissertação [mestrado em psicologia] - Universidade Federal de Juiz de Fora;2014.

9. Leão IS, Eulálio MC. Velhice e atividade profissional: um estudo sobre qualidade de vida. In: Psicologia da Saúde: teoria, intervenção e pesquisa. Campina Grande:Universidade Estadual da Paraíba;2011. p. 199-216.

10. Cañabate P, Martínez G, Rosende-Roca M, et al. Social Representation of Dementia: An Analysis of 5,792 Consecutive Cases Evaluated in a Memory Clinic. J Alzheimers Dis. 2017;58(4):10991108. doi: 10.3233/JAD-161119.

11. Torres TL, Camargo BV, Boulsfield AB, et al. Social representations and normative beliefs of aging. Ciênc Saúde Coletiva. dezembro de 2015;20(12):3621-30.

12. Lourenço RA, Moreira VLG, Banhato EFC, et al. Prevalence of frailty and associated factors in a community-dwelling older people cohort living in Juiz de Fora, Minas Gerais, Brazil: Fibra-JF Study. Ciênc Saúde Coletiva. 2019;24(1):35-44.

13. Censo demográfico 2010: características gerais da população, religião e pessoas com deficiência. Rio de Janeiro:Instituto Brasileiro de Geografia e Estatística - IBGE;2010.

14. Angelini V, Cavapozzi D, Corazzini L, et al. Age, Health and Life Satisfaction Among Older Europeans. Soc Indic Res. 
Elaine A. Moura e cols. • Satisfação com a vida de idosos aposentados

2012;105(2):293-308.

15. Mármora CHC, Moura EA. Proposições acerca de um novo paradigma da senescência baseada na percepção do envelhecimento. In: Pesquisas em desenvolvimento humano e educação. Curitiba: CRV; 2016. p. 337-51.

16. Pereira KCR, Alvarez AM, Traebert JL. Contribution of socio-demographic conditions for the perception of quality of life in the elderly. Rev Bras Geriatr e Gerontol. 2011;14(1):85-95.

17. Pilger C, Menon MH, Mathias TA de F. Socio-demographic and health characteristics of elderly individuals: support for health services. Rev Lat Am Enfermagem. 2011;19(5):1230-8.
18. Martins SC. Relato de uma experiência de ensino de língua italiana para a terceira idade: desconstruindo concepções e arquitetando uma nova visão de mundo. Trab Em Linguística Apl. 2017;56(1):117-37.

19. Silva RS, Rodrigues ND. Saúde Emocional: a importância de Planejar a Aposentadoria. Ens E Ciênc C Biológicas Agrár e Saúde. 2016;20(2):116-21.

20. Barros A, Muniz TS. O trabalhador idoso no mercado de trabalho do capitalismo contemporâneo. Cad Grad - Ciênc Humanas e Sociais - UNIT - Alagoas. 2014;2(1):103-16. 